\title{
ERGODICITY AND DIFFERENCES OF FUNCTIONS ON SEMIGROUPS
}

\author{
BOLIS BASIT and A. J. PRYDE
}

(Received 18 July 1997)

Communicated by E. N. Dancer

\begin{abstract}
Iseki [11] defined a general notion of ergodicity suitable for functions $\varphi: J \rightarrow X$ where $J$ is an arbitrary abelian semigroup and $X$ is a Banach space. In this paper we develop the theory of such functions, showing in particular that it fits the general framework established by Eberlein [9] for ergodicity of semigroups of operators acting on $X$. Moreover, let $\mathscr{A}$ be a translation invariant closed subspace of the space of all bounded functions from $J$ to $X$. We prove that if $\mathscr{A}$ contains the constant functions and $\varphi$ is an ergodic function whose differences lie in $\mathscr{A}$ then $\varphi \in \mathscr{A}$. This result has applications to spaces of sequences facilitating new proofs of theorems of Gelfand and Katznelson-Tzafriri [12]. We also obtain a decomposition for the space of ergodic vectors of a representation $T: J \rightarrow L(X)$ generalizing results known for the case $J=\mathbb{Z}^{+}$. Finally, when $J$ is a subsemigroup of a locally compact abelian group $G$, we compare the Iseki integrals with the better known Cesàro integrals.
\end{abstract}

1991 Mathematics subject classification (Amer. Math. Soc.): primary 43A60; secondary 47A 10, 47D03, $28 \mathrm{~B} 05$.

Key'ords and phrases: Ergodic, semigroup, differences, Beurling spectrum, invariant means, system of invariant integrals.

\section{Introduction}

In a successful attempt to unify and extend the growing collection of ergodic theorems, Eberlein [9] introduced systems of almost invariant integrals for semigroups of continuous linear transformations on locally convex spaces. A semigroup possessing such a system he called ergodic, and for such semigroups he proved a very general mean ergodic theorem ([9, Theorem 3.1]). Since that time many more ergodic theorems have appeared and many have been revealed as special cases of Eberlein's classical theorem. See for example [17].

(C) 1998 Australian Mathematical Society $0263-6115 / 98 \$ A 2.00+0.00$ 
In a different direction, Iseki [11] introduced the notion of ergodicity of functions $\varphi: J \rightarrow X$ where $J$ is a semigroup and $X$ is a locally convex space. With it he was able to show that every such function which is almost periodic in the sense of Maak is necessarily ergodic.

Ruess and Summers [18] considered asymptotically almost periodic functions $\varphi$ : $\mathbb{R}^{+} \rightarrow X$. They showed that if the indefinite integral $\Phi$ of $\varphi$ is weakly almost periodic in the sense of Eberlein, then $\Phi$ is asymptotically almost periodic. Subsequently Basit [3] observed that weak almost periodicity could be replaced by the more general property of ergodicity, that is the Cesàro integrals of $\Phi$ converge uniformly to a constant. Moreover, he replaced asymptotically almost periodic functions by large classes of functions. Ruess and Phóng [16] independently obtained some of these results.

Basit also observed that the integral problem discussed above is closely related to the difference problem: if $\varphi \in C_{b}(J, X)$ and $\Delta_{1} \varphi \in \mathscr{A} \subseteq C_{b}(J, X)$ for all $t \in J$, find conditions that ensure $\varphi \in \mathscr{A}$. Basit investigated this problem for the cases $J=\mathbb{B}^{+}$ or $\mathbb{R}$ and gave applications to the solutions of certain integro-differential difference equations [3] and to the abstract Cauchy problem [4]. Once again ergodicity of $\varphi$ played an important role.

In the present paper we develop the theory of (Iseki) ergodic functions $\varphi: J \rightarrow X$ where $J$ is an arbitrary semigroup and $X$ is a Banach space. For the sake of simplicity and clarity, we restrict ourselves to the case of abelian $J$. In particular, we show how this theory fits into the framework established by Eberlein. Our main result concerns the difference problem and its relationship with ergodicity. This is in Section 2.

In Section 3 we apply our results to spaces of sequences. Among other things we obtain new proofs of theorems of Gelfand and Katznelson-Tzafriri on power bounded elements of Banach algebras. Section 4 deals with representations of semigroups on Banach spaces. We obtain a decomposition for the subspace of ergodic vectors generalizing known results for the case $J=\mathbb{Z}^{+}$.

Finally, in section 5 we exhibit a large class of semigroups $J$ for which one can take limits of Cesàro integrals of functions $\varphi$ in $C_{u b}(J, X)$. We show that these limits, when they exist, are identical to the Iseki means. Similarly, when $G$ is a locally compact abelian group, we show that the means studied by Argabright [2] and Datry and Muraz [7] for $\varphi \in C_{b}(G, X)$ are identical to the Iseki means. We conclude by giving a simple condition on the Beurling spectrum of a function $\varphi \in C_{u b}(G, X)$ that ensures $\varphi$ is ergodic. 


\section{Ergodicity}

Throughout this paper, $J$ will denote an abelian semigroup and $X$ a Banach space over $\mathbb{R}$ or $\mathbb{C}$. By $B(J, X)$ we denote the space of bounded functions $\varphi: J \rightarrow X$, endowed with the norm $\|\varphi\|_{\infty}=\sup _{t \in J}\|\varphi(t)\|$. For such a function, $\varphi_{s}$ and $\Delta_{s} \varphi$ will denote the translate and difference by $s$ of $\varphi$, defined by $\varphi_{s}(t)=\varphi(t+s)$ and $\Delta_{s} \varphi=\varphi_{s}-\varphi$ for $s, t \in J$. The closed subspaces of $B(J, X)$ consisting of continuous and uniformly continuous functions respectively are denoted $C_{b}(J, X)$ and $C_{u b}(J, X)$. We will use the same symbol, say $x$, for an element of $X$ and for the function in $B(J, X)$ taking the constant value $x$.

Following Iseki [11, I] we say that a function $\varphi: J \rightarrow X$ is ergodic if $\varphi \in B(J, X)$ and there exists $M_{\varphi} \in X$ such that for each $\varepsilon>0$ there are elements $t_{1}, \ldots, t_{n} \in J$ with $\left\|(1 / n) \sum_{i=1}^{n}\left(\varphi_{t_{i}}-M_{\varphi}\right)\right\|_{\infty}<\varepsilon$. The element $M_{\varphi}$, clearly unique, is called the (Iseki) mean of $\varphi$ and the class of all such ergodic functions is denoted $E(J, X)$. We define $M: E(J, X) \rightarrow X$ by $M(\varphi)=M_{\varphi}$.

PROPOSITION 2.1. The space $E(J, X)$ is a translation invariant closed subspace of $B(J, X)$ containing all the constant functions. Moreover, $M: E(J, X) \rightarrow X$ is a bounded linear map.

Proof. Let $\varphi, \psi \in E(J, X)$. By the definition of ergodicity, for each $\varepsilon>0$ there exist elements $s_{1}, \ldots, s_{m}, t_{1}, \ldots, t_{n} \in J$ such that $\left\|(1 / m) \sum_{i=1}^{m}\left(\varphi_{s_{i}}-M_{\varphi}\right)\right\|_{\infty}<\varepsilon$ and $\left\|(1 / n) \sum_{j=1}^{n}\left(\psi_{t_{j}}-M_{\psi}\right)\right\|_{\infty}<\varepsilon$. Since $\left\|\varphi_{t}\right\|_{\infty} \leq\|\varphi\|_{\infty}$ for all $t \in J$, we obtain $\left\|(1 / n m) \sum_{i=1}^{m} \sum_{j=1}^{n}\left(\varphi_{s_{i}+t_{j}}+\psi_{s_{i}+t_{j}}-M_{\varphi}-M_{\psi}\right)\right\|_{\infty}<2 \varepsilon$. Hence $\varphi+\psi \in E(J, X)$ and $M(\varphi+\psi)=M(\varphi)+M(\psi)$. The rest of the proposition is proved similarly.

The following result shows that there are many ergodic functions. Further examples will be provided later.

PRoposition 2.2. If $\varphi \in B(J, X)$ and $s \in J$ then $\Delta_{s} \varphi \in E(J, X)$ and $M\left(\Delta_{s} \varphi\right)=$ 0 .

PROOF. Given $\varepsilon>0$, choose $n \in \mathbb{N}$ such that $\|(1 / n) \varphi\|_{\infty}<\varepsilon / 2$. Since $\left(\Delta_{s} \varphi\right)_{t}=$ $\Delta_{s+t} \varphi-\Delta_{t} \varphi$, we have $\left\|(1 / n) \sum_{j=1}^{n}\left(\Delta_{s} \varphi\right)_{j s}\right\|_{\infty}<\varepsilon$. This proves the proposition.

The following alternative characterization of ergodic functions will be useful. For this we set $\mathscr{F}(J)=\{F \subseteq J:|F|<\infty\}$ where $|F|$ is the cardinality of $F$. Then $\mathscr{F}(J)$ becomes a directed set if we define $F_{1} \leq F_{2}$ whenever there exists $F \in \mathscr{F}(J)$ such that $F_{2}=F_{1}+F$.

Proposition 2.3. Let $\varphi \in B(J, X)$. Then $\varphi \in E(J, X)$ if and only if there exists $y \in X$ such that $\lim _{F \in \mathscr{F}_{(J)}}\left((1 /|F|) \sum_{t \in F} \varphi_{t}\right)=y$. In this case, $y=M_{\varphi}$. 
Proof. Let $\varphi \in E(J, X)$. For each $\varepsilon>0$ there is a set $F_{\varepsilon} \in \mathscr{F}(J)$ such that $\left\|\left(1 /\left|F_{\varepsilon}\right|\right) \sum_{t \in F_{\varepsilon}}\left(\varphi_{t}-M_{\varphi}\right)\right\|_{\infty}<\varepsilon$. If $F \in \mathscr{F}(J)$ satisfies $F \geq F_{\varepsilon}$, that is $F=F_{\varepsilon}+H$ for some $H \in \mathscr{F}(J)$, then

$$
\left\|\frac{1}{|F|} \sum_{u \in F}\left(\varphi_{u}-M_{\varphi}\right)\right\|_{\infty}=\left\|\frac{1}{\left|F_{\varepsilon}\right|} \cdot \frac{1}{|H|} \sum_{t \in F_{\varepsilon}} \sum_{s \in H}\left(\varphi_{t+s}-M_{\varphi}\right)\right\|_{\infty}<\varepsilon,
$$

showing that $\lim _{F \in \mathscr{F}_{(J)}}(1 /|F|) \sum_{t \in F} \varphi_{t}=M_{\varphi}$. The converse is clear.

Our next task is to set Iseki ergodicity in the framework of Eberlein. For this, let $\mathscr{S}$ be a sub-semigroup under composition of the Banach algebra $L(E)$ of all bounded operators $A: E \rightarrow E$ where $E$ is a Banach space. The orbit of $x \in E$ under $\mathscr{S}$ is $\operatorname{orb}_{\mathscr{S}}(x)=\{S x: S \in \mathscr{S}\}$. A net $\left(A_{\alpha}\right)_{\alpha \in \Lambda}$ in $L(E)$ is called a system of invariant integrals for $\mathscr{S}$ if

(2.1) $A_{\alpha} x \in \overline{\operatorname{co}} \operatorname{orb}_{\mathscr{S}}(x)$ for all $x \in E$ and $\alpha \in \Lambda$,

(2.2) $\sup _{\alpha \in \Lambda}\left\|A_{\alpha}\right\|<\infty$,

(2.3) $\lim _{\alpha \in \Lambda}\left\|\left(A_{\alpha} S-A_{\alpha}\right) x\right\|=\lim _{\alpha \in \Lambda}\left\|\left(S A_{\alpha}-A_{\alpha}\right) x\right\|=0$ for all $x \in E$ and $S \in \mathscr{S}$.

If (2.1), (2.2) hold but (2.3) only holds at $x_{0} \in E$ then we say $\left(A_{\alpha}\right)$ is a system of invariant integrals for $\mathscr{S}$ at $x_{0}$.

For $\varphi \in B(J, X), F \in \mathscr{F}(J)$ and $s \in J$, define $R_{F} \varphi=(1 /|F|) \sum_{t \in F} \varphi_{l}$, interpreted as 0 if $F=\emptyset$, and $R_{s}=R_{\{s\}}$. Hence $R_{F}, R_{s} \in L(E)$ where $E=B(J, X)$.

PROPOSITION 2.4. The net $\left(R_{F}\right)_{F \in \mathscr{F}(J)}$ is a system of invariant integrals for the translation semigroup $\mathscr{R}=\left\{R_{s}: s \in J\right\}$.

Proof. For $\varphi \in B(J, X),\left(R_{F} R_{s}-R_{F}\right) \varphi=R_{F}\left(\Delta_{s} \varphi\right)$. By Proposition 2.2, $M\left(\Delta_{s} \varphi\right)=0$ and so by Proposition 2.3, $\lim _{F \in \mathscr{F}_{(J)}}\left(R_{F} R_{s}-R_{F}\right) \varphi=0$. Hence (2.3) follows, and (2.1), (2.2) are obvious.

By Eberlein's mean ergodic theorem [9, Theorem 3.1] we have immediately

COROLLARY 2.5. For $\varphi \in B(J, X)$ the following are equivalent

(1) $\varphi \in E(J, X)$ and $M(\varphi)=y$,

(2) the net $\left(R_{F} \varphi\right)_{F \in \mathscr{F}(J)}$ converges to $y$,

(3) some subnet of $\left(R_{F} \varphi\right)_{F \in \mathscr{F}(J)}$ converges weakly to $y$,

(4) $y \in \overline{\operatorname{co}} \operatorname{orb}_{\mathscr{R}}(\varphi)$ with y a constant function. 
Recall that the space $W(J, X)$ of Eberlein weakly almost periodic functions consists of the bounded functions $\varphi: J \rightarrow X$ for which $\operatorname{orb}_{\mathscr{R}}(\varphi)$ is weakly relatively compact. From Corollary 2.5 we obtain

COROLlaRY 2.6. $W(J, X)$ is a closed linear subspace of $E(J, X)$.

Note that $M: E(J, X) \rightarrow X$ is a (translation) invariant mean in the sense of [6, p.79] for scalar $X$ and [21] for general $X$. The latter proved the existence of an invariant mean on $W(J, X)$ for certain non-abelian semigroups $J$ [21, Theorem 8.7]. However, the invariant means in these references are not given explicitly.

To conclude this section we prove our main result for ergodic functions. With the additional assumption that $\mathscr{A}$ contains the constant functions, this theorem provides a solution of the difference problem.

THEOREM 2.7. Let $\mathscr{A}$ be a translation invariant closed subspace of $B(J, X)$. If $\varphi \in E(J, X)$ and $\Delta_{l} \varphi \in \mathscr{A}$ for all $t \in J$, then $\varphi-M(\varphi) \in \mathscr{A}$.

PROOF. For each non-empty $F \in \mathscr{F}(J)$ we have $\varphi-R_{F} \varphi=-(1 /|F|) \sum_{t \in F} \Delta_{t} \varphi \in$ $\mathscr{A}$. The theorem follows from Corollary 2.5 by taking the limit over $F$ in $\mathscr{F}(J)$.

\section{Sequence spaces}

In this section we give some applications of our results to spaces of sequences. Here we take $J=\mathbb{Z}, \mathbb{Z}^{+}$or $\mathbb{Z}^{-}$and use the condition

(3.1) $\mathscr{A}$ is a closed subspace of $B(J, X)$ such that $\left.\psi_{t}\right|_{J} \in \mathscr{A}$ whenever $\psi \in B(\mathbb{Z}, X)$, $t \in \mathbb{Z}$ and $\left.\psi\right|_{J} \in \mathscr{A}$.

Examples of such subspaces $\mathscr{A}$ include $E(J, X)$, the space $C_{0}(J, X)$ of functions convergent to 0 at infinity, the space $A P(\mathbb{Z}, X)$ of almost periodic functions and the space $\operatorname{WAP}(J, X)$ of Eberlein weakly almost periodic functions.

Following [3, Definition 4.1.2] we define the spectrum with respect to $\mathscr{A}$ of a function $\varphi \in B(\mathbb{Z}, X)$ by $\operatorname{sp}_{\mathscr{A}}(\varphi)=\left\{\gamma \in \widehat{\mathbb{Z}}: \hat{f}(\gamma)=0\right.$ for all $\left.f \in I_{\mathscr{A}}(\varphi)\right\}$ where $\widehat{\mathbb{Z}}$ is the (unitary) character group of $\mathbb{Z}, \hat{f}: \widehat{\mathbb{Z}} \rightarrow \mathbb{C}$ is the Fourier transform of $f$, and $I_{\mathscr{A}}(\varphi)=\left\{f \in L^{1}(\mathbb{Z}):\left.(\varphi * f)\right|_{J} \in \mathscr{A}\right\}$.

The following proposition is well-known for the case $\mathscr{A}=\{0\}$ and $J=\mathbb{Z}$, in which case $\operatorname{sp}_{\mathscr{A}}(\varphi)=\operatorname{sp}(\varphi)$, the Beurling spectrum of $\varphi$.

Proposition 3.1. Let $\varphi, \psi \in B(\mathbb{Z}, X), f \in L^{1}(\mathbb{Z}), \gamma \in \widehat{\mathbb{Z}}$ and $\mathscr{A}$ satisfy condition (3.1).

(i) $\operatorname{sp}_{\mathscr{A}}(\varphi)=\operatorname{sp}_{\mathscr{A}}\left(\varphi_{t}\right)$ for all $t \in \mathbb{Z}$. 
(ii) $\operatorname{sp}_{\mathscr{A}}(\varphi * f) \subseteq \operatorname{sp}_{\mathscr{A}}(\varphi) \cap \operatorname{supp}(\hat{f})$.

(iii) $\operatorname{sp}_{\mathscr{A}}(\varphi+\psi) \subseteq \operatorname{sp}_{\mathscr{A}}(\varphi) \cup \operatorname{sp}_{\mathscr{A}}(\psi)$.

(iv) $\operatorname{sp}_{\mathscr{A}}(\gamma \varphi)=\gamma+\operatorname{sp}_{\mathscr{A}}(\varphi)$.

(v) $\operatorname{sp}_{\mathscr{A}}(\varphi)=\emptyset$ if and only if $\left.\varphi\right|_{J} \in \mathscr{A}$.

PROOF. The arguments are the same as for the Beurling spectrum. See for example [8, part II, p.988] or [5]. We present a proof for (v). If $\left.\varphi\right|_{J} \in \mathscr{A}$ then by (3.1), $\left.\varphi_{t}\right|_{J} \in \mathscr{A}$ for all $t \in \mathbb{Z}$. Hence for $f \in L^{1}(\mathbb{Z}),\left.(\varphi * f)\right|_{J}=\left.\sum_{n \in \mathbb{Z}} f(n) \varphi_{-n}\right|_{J} \in \mathscr{A}$. So $I_{\mathscr{A}}(\varphi)=L^{1}(\mathbb{Z})$ and $\operatorname{sp}_{\mathscr{A}}(\varphi)=\emptyset$. Conversely, if $\operatorname{sp}_{\mathscr{A}}(\varphi)=\emptyset$ then $I_{\mathscr{A}}(\varphi)=L^{1}(\mathbb{Z})$. Choose $f_{n} \in L^{1}(\mathbb{Z})$ such that $\varphi * f_{n} \rightarrow \varphi$ in $B(\mathbb{Z}, X)$. Since $f_{n} \in I_{\mathscr{A}}(\varphi),\left.\left(\varphi * f_{n}\right)\right|_{J} \in$ $\mathscr{A}$ and since $\mathscr{A}$ is closed, $\left.\varphi\right|_{J} \in \mathscr{A}$.

In the sequel we denote the elements of $\widehat{\mathbb{Z}}$ by $\gamma_{\lambda}$ or $\lambda$, where $\lambda \in \mathbb{U}$ the circle group and $\gamma_{\lambda}(n)=\lambda^{n}$ for $n \in \mathbb{Z}$. Hence $\gamma_{1}$ or 1 is the unit in $\widehat{\mathbb{Z}}$.

Proposition 3.2. Suppose $\mathscr{A}$ satisfies (3.1), $\varphi \in B(J, X),\left.\varphi\right|_{\jmath} \in E(J, X)$ and $\operatorname{sp}_{\mathscr{A}}(\varphi) \subseteq\{1\}$. Then $\left.\varphi\right|_{J}-M\left(\left.\varphi\right|_{J}\right) \in \mathscr{A}$.

PROOF. By Wiener's tauberian theorem $[15,7.2 .5]$ the condition $\operatorname{sp}_{\mathscr{A}}(\varphi) \subseteq\{1\}$ is equivalent to $I_{\mathscr{A}}(\varphi) \supseteq\left\{f \in L^{1}(\mathbb{Z}): \hat{f}(1)=0\right\}$. For $t \in \mathbb{Z}, g \in L^{1}(\mathbb{Z})$ and $\lambda \in \mathbb{T}$ we have $\left(\Delta_{t} g\right)(\lambda)=\left(\gamma_{\lambda}(t)-1\right) \hat{g}(\lambda)$. Hence $\Delta_{t} g \in I_{\mathscr{A}}(\varphi)$. In other words, $\left.\left(\Delta_{t} \varphi * g\right)\right|_{J}=\left.\left(\varphi * \Delta_{t} g\right)\right|_{J} \in \mathscr{A}$. Setting $g=\chi_{[0]}$, the characteristic function of $\{0\}$ in $\mathbb{Z}$ we have $\Delta_{t} \varphi=\Delta_{t} \varphi * g$ and so $\left.\Delta_{t} \varphi\right|_{J} \in \mathscr{A}$. By Theorem 2.7, $\left.\varphi\right|_{J}-M\left(\left.\varphi\right|_{J}\right) \in \mathscr{A}$.

As a consequence we have the following application of spectra to the difference problem.

THEOREM 3.3. Suppose $\mathscr{A}$ satisfies (3.1) and $\varphi \in B(\mathbb{Z}, X)$. Then $\operatorname{sp}_{\mathscr{A}}(\varphi) \subseteq\{1\}$ if and only if $\left.\Delta_{t} \varphi\right|_{J} \in \mathscr{A}$ for all $t \in J$.

PRoOF. Let $\left.\Delta_{t} \varphi\right|_{J} \in \mathscr{A}$ for all $t \in J$. If $g \in L^{1}(\mathbb{Z})$ then by (3.1), $\left.\left(\varphi * \Delta_{t} g\right)\right|_{J}=$ $\left.\sum_{n \in \mathbb{Z}} g(n)\left(\Delta_{t} \varphi\right)_{-n}\right|_{J} \in \mathscr{A}$. So $I_{\mathscr{A}}(\varphi) \supseteq\left\{\Delta_{t} g: t \in J, g \in L^{1}(\mathbb{Z})\right\}$. But $\left(\Delta_{t} g\right)(\lambda)=$ $\left(\gamma_{\lambda}(t)-1\right) \hat{g}(\lambda)$ is zero for all $t \in J$ and $g \in L^{1}(\mathbb{Z})$ only when $\lambda=1$. So $\operatorname{sp}_{\mathscr{A}}(\varphi) \subseteq$ $\{1\}$. Conversely, let $\operatorname{sp}_{\mathscr{A}}(\varphi) \subseteq\{1\}$. By Proposition $2.2,\left.\Delta_{,} \varphi\right|_{J} \in E(J, X)$ and $M\left(\left.\Delta_{t} \varphi\right|_{J}\right)=0$ for each $t \in J$. By Proposition 3.2, $\left.\Delta_{t} \varphi\right|_{J} \in \mathscr{A}$.

In order to apply Theorem 3.3, we first prove the following result. In it, $\sigma(x)$ denotes the Banach algebra spectrum of $x$.

THEOREM 3.4. Let $X$ be a unital Banach algebra. Suppose $\mathscr{A} \subseteq B(J, X)$ satisfies (3.1) and in addition $y \mathscr{A} \subseteq \mathscr{A}$ for all $y \in X$. Let $\varphi: \mathbb{Z} \rightarrow X$ be a bounded solution of the recurrence equation $\varphi(n+1)=x \varphi(n)+\psi(n)$ for some $x \in X$ and $\psi \in C_{b}(\mathbb{Z}, X)$. If $\left.\psi\right|_{J} \in \mathscr{A}$ then $\operatorname{sp}_{\mathscr{A}}(\varphi) \subseteq \sigma(x) \cap \mathbb{T}$. 
PRoOF. Let $\lambda_{0} \in \mathbb{T} \backslash \sigma(x)$. Choose $\delta>0$ such that $B_{\delta}\left(\lambda_{0}\right)=\left\{\lambda \in \mathbb{C}:\left|\lambda-\lambda_{0}\right|<\right.$ $\delta\} \subseteq \mathbb{C} \backslash \sigma(x)$. Take $f \in L^{1}(\mathbb{Z})$ with $\hat{f}\left(\lambda_{0}\right)=1$ and $\operatorname{supp}(\hat{f}) \subseteq B_{\delta / 4}\left(\lambda_{0}\right)$. Let $\xi=\varphi * f$. It suffices to prove $\left.\xi\right|_{J} \in \mathscr{A}$, for then $f \in I_{\mathscr{A}}(\varphi)$ and $\lambda_{0} \notin \operatorname{sp}_{\mathscr{A}}(\varphi)$.

To do this, let $g \in L^{1}(\mathbb{Z})$ be such that $\hat{g}(\lambda)=1$ for $\lambda \in B_{\delta / 2}\left(\lambda_{0}\right) \cap \mathbb{J}, \operatorname{supp}(\hat{g}) \subseteq$ $B_{\delta}\left(\lambda_{0}\right)$ and $\hat{g} \in C^{1}(\mathbb{T})$. Define $h: \mathbb{T} \rightarrow X$ by $\hat{h}(\lambda)=\hat{g}(\lambda)(\lambda e-x)^{-1}$, interpreted as 0 outside $B_{\delta}\left(\lambda_{0}\right)$, where $e$ is the unit in $X$. Then $\hat{h} \in C^{\mathrm{l}}(\mathbb{J}, X)$ so $\hat{h}(\lambda)=$ $\sum_{n=-\infty}^{\infty} h(n) \lambda^{-n}$ for some $h \in L^{1}(\mathbb{Z}, X)$ with $h(n) x=x h(n)$ for all $n \in \mathbb{Z}$. Moreover, if $\eta_{\lambda}(n)=\gamma_{\lambda}(n+1) e-\gamma_{\lambda}(n) x$, where $\gamma_{\lambda}(n)=\lambda^{n}$ and $\lambda \in B_{\delta / 2}\left(\lambda_{0}\right) \cap \mathbb{T}$, then $h * \eta_{\lambda}=\gamma_{\lambda}$. Indeed,

$$
\begin{aligned}
h * \eta_{\lambda}(n) & =\sum_{j} h(j)\left(\lambda^{n+1-j} e-\lambda^{n-j} x\right)=\lambda^{n}(\lambda e-x) \sum_{j} h(j) \lambda^{-j} \\
& =\lambda^{n}(\lambda e-x) \hat{g}(\lambda)(\lambda e-x)^{-1}=\lambda^{n} .
\end{aligned}
$$

Now $\xi=\varphi * f \in B(\mathbb{Z}, X)$ and $\operatorname{sp}(\xi) \subseteq \operatorname{supp}(\hat{f}) \subseteq B_{\delta / 4}\left(\lambda_{0}\right)$, so there is a sequence of trigonometric polynomials $\pi_{m} \in B(\mathbb{Z}, X)$ converging pointwise to $\xi$ and with $\operatorname{sp}\left(\pi_{n}\right) \subseteq B_{\delta / 2}\left(\lambda_{0}\right)$. Let $\eta_{m}(n)=\pi_{m}(n+1) e-x \pi_{m}(n)$. Then $h * \eta_{m}=\pi_{m}$.

From the recurrence equation, $\eta_{m}(n) \rightarrow \xi(n+1)-x \xi(n)=\psi * f(n)$ for each $n \in \mathbb{Z}$. Hence $\xi=h * \psi * f$. Since $\xi=\sum_{n \in \mathbb{Z}} h(n)(\psi * f)_{-n}$ and $y \mathscr{A} \subseteq \mathscr{A}$ for each $y \in X$, it follows from (3.1) that $\left.\xi\right|_{J} \in \mathscr{A}$ as required.

As a consequence we easily obtain the following two results. The first was proved by Gelfand (see [12]) and the second by Katznelson and Tzafriri [12]. Recall that an element $x$ of a unital Banach algebra $X$ is called power bounded if $\left\{x^{n}: n \in \mathbb{Z}^{+}\right\}$is bounded and doubly power bounded if $\left\{x^{n}: n \in \mathbb{Z}\right\}$ is bounded.

COROLLARY 3.5. Let $x$ be a doubly power bounded element of a unital Banach algebra $X$. If $\sigma(x)=\{1\}$ then $x=e$.

PROOF. We may apply Theorem 3.4 with $\mathscr{A}=\{0\}, J=\mathbb{Z}, \psi=0$ and $\varphi(n)=x^{n}$. So $\operatorname{sp}(\varphi) \subseteq \sigma(x) \cap \mathbb{Z}=\{1\}$. By Theorem 3.3, $\Delta_{t} \varphi=0$ for all $t \in \mathbb{Z}$ and hence $x=e$.

COROLLARY 3.6. Let $x$ be a power bounded element of a unital Banach algebra X. If $\sigma(x) \cap \mathbb{T} \subseteq\{1\}$ then $\left\|x^{n+1}-x^{n}\right\| \rightarrow 0$ as $n \rightarrow \infty$.

PROOF. Apply Theorem 3.4 with $\mathscr{A}=C_{0}(J, X), J=\mathbb{Z}^{+}$and $\varphi, \psi$ as follows. For $n \geq 0$ set $\varphi(n)=x^{n}, \psi(n)=0$ and for $n<0$ set $\varphi(n)=e, \psi(n)=e-x$. So $\operatorname{sp}_{\mathscr{A}}(\varphi) \subseteq\{1\}$ and by Theorem 3.3, $\left.\Delta_{t} \varphi\right|_{J} \in \mathscr{A}$ for all $t \in J$. This gives the corollary.

In a subsequent paper we will use ergodicity and the difference problem to obtain generalizations of these last two results. 


\section{Ergodic vectors of representations}

Throughout this section $J$ will denote an abelian semigroup and $T: J \rightarrow L(X)$ a representation. That is, $T$ is a semigroup homomorphism mapping $J$ into the semigroup under composition $L(X)$. The dual representation $T^{*}: J \rightarrow L\left(X^{*}\right)$ is defined by $\left\langle x, T^{*}(t) \varphi\right\rangle=\langle T(t) x, \varphi\rangle$ for $x \in X, t \in J$ and $\varphi \in X^{*}$.

The space of fixed points of $T$ is $N=N(T)=\bigcap_{t \in J} \operatorname{ker}(T(t)-I)$ and its complementary space is $R=R(T)=\operatorname{span}\{T(s) x-x: x \in X, s \in J\}$. The closure of $R$ is denoted $\bar{R}=\bar{R}(T)$. The set of ergodic vectors of $T$ is $X_{\mathrm{erg}}=X_{\mathrm{erg}}(T)=\{x \in$ $X: T(\cdot) x \in E(J, X)\}$.

Next let $T(J)$ be the range of $T$ in $L(X)$ and for $F \in \mathscr{F}(J)$ define $T_{F} \in L(X)$ by $T_{F} x=(1 /|F|) \sum_{t \in F} T(t) x$, again interpreted as 0 if $F=\emptyset$. Finally, the orbit under $T$ of an element $x \in X$ is $\operatorname{orb}_{T}(x)=\operatorname{orb}_{T(J)}(x)$.

PROPOSITION 4.1. If $T: J \rightarrow L(X)$ is a representation and $\operatorname{orb}_{T}(x)$ is bounded for some $x \in X$, then the set $\left(T_{F}\right)_{F \in \mathscr{F}(J)}$ is a system of invariant integrals for the semigroup $T(J)$ at $x$.

Proof. Let $s \in J$. The function $T(\cdot) x: J \rightarrow X$ is bounded and hence by Proposition 2.2, $\Delta_{s} T(\cdot) x \in E(J, X)$ and $M\left(\Delta_{s} T(\cdot) x\right)=0$. By Corollary 2.5, $\lim _{F} R_{F} \Delta_{s} T(\cdot) x=0$ and in particular $\lim _{F}\left\|R_{F} \Delta_{s} T(t) x\right\|=0$ for each $t \in J$. But $R_{F} \Delta_{s} T(t) x=\left(R_{F+1} T(s)-R_{F+1}\right) x$ and so $\lim _{F}\left\|\left(R_{F} T(s)-R_{F}\right) x\right\|=0$. Condition (2.3) follows for this $x$. Since (2.1) and (2.2) are clear the proposition is proved.

COROLLARY 4.2. If $T: J \rightarrow L(X)$ is a representation and $\operatorname{orb}_{T}(x)$ is bounded for some $x \in X$ then the following are equivalent

(i) $x \in X_{\mathrm{erg}}(T)$ and $M(T(\cdot) x)=y$,

(ii) $\left(T_{F} x\right)_{F \in \mathscr{F}(J)}$ converges to $y$,

(iii) some subnet of $\left(T_{F} x\right)_{F \in \mathscr{F}(J)}$ converges weakly to $y$.

(iv) $y \in N(T) \cap \overline{\operatorname{co}} \operatorname{orb}_{T}(x)$.

PROOF. By Eberlein's mean ergodic theorem (see Theorem 3.1 in [9] and the remark following it) we conclude that (ii), (iii) and (iv) are equivalent. Let $\kappa=\sup \{\|z\|$ : $\left.z \in \operatorname{orb}_{T}(x)\right\}$. Then for each $t \in J$ and $F \in \mathscr{F}(J)$ we have $\left\|T_{F+t} x-y\right\|=$ $\left\|R_{F} T(t) x-y\right\| \leq\left\|R_{F} T(\cdot) x-y\right\|_{\infty} \leq \kappa\left\|T_{F} x-y\right\|$. Hence $\left(T_{F} x\right) \rightarrow y$ in $X$ if and only if $\left(R_{F} T(\cdot) x\right) \rightarrow y$ in $B(J, X)$. By Corollary 2.5, (ii) is equivalent to (i).

Proposition 4.3. If $T: J \rightarrow L(X)$ is a bounded representation, then $X_{\mathrm{erg}}$ is a closed linear subspace of $X$. Moreover, $X_{\mathrm{erg}}=N \oplus \bar{R}$. 
ProOf. Since $E(J, X)$ is a linear space, so too is $X_{\text {erg. }}$. The closedness of $X_{\text {erg }}$ follows from the boundedness of $T$ and the closedness of $E(J, X)$ in $B(J, X)$. If $x \in N$ then $T(t) x=x$ for all $t \in J$. Hence $T(\cdot) x \in E(J, X)$ and $M(T(\cdot) x)=x$, showing $N \subseteq X_{\text {erg. }}$. If $z \in R$ then there exist $t_{1}, \ldots, t_{n} \in J$ and $x_{1}, \ldots, x_{n} \in X$ such that $z=\sum_{j=1}^{n}\left(T\left(t_{j}\right) x_{j}-x_{j}\right)$. Hence $T(\cdot) z=\sum_{j=1}^{n} \Delta_{t_{j}} T(\cdot) x_{j}$. By Proposition 2.2, $T(\cdot) z \in E(J, X)$ and $M(T(\cdot) z)=0$. By Proposition 2.1, the same is true for $z \in \bar{R}$. Hence $\bar{R} \subseteq X_{\text {erg }}$ and moreover, $N \cap \bar{R}=\{0\}$.

Finally we show $X_{\mathrm{erg}} \subseteq N+\bar{R}$. If $y \in X_{\mathrm{erg}}$ then by Corollary $4.2, M(T(\cdot) y) \in N$. Setting $z=y-M(T(\cdot) y)$ we show $z \in \bar{R}$. Indeed, for each $\varepsilon>0$ there exist $t_{1}, \ldots, t_{n} \in J$ such that $\left\|(1 / n) \sum_{j=1}^{n}\left[T(t) T\left(t_{j}\right) y-M(T(\cdot) y)\right]\right\|<\varepsilon$ for all $t \in J$. Now $z_{\varepsilon}=(1 / n) \sum_{j=1}^{n}\left[z-T\left(t+t_{j}\right) z\right] \in R$ and $\left\|z-z_{\varepsilon}\right\|<\varepsilon$, so $z \in \bar{R}$. Hence $y \in N+\bar{R}$ and the proposition is proved.

The following two results provide examples of ergodic vectors.

COROLLARY 4.4. Let $T: J \rightarrow L(X)$ be a representation and $x \in X$. If $\operatorname{orb}_{T}(x)$ is weakly relatively compact then $x \in X_{\mathrm{erg}}(T)$.

PROOF. Since $\operatorname{orb}_{T}(x)$ is weakly relatively compact, it is bounded and by Proposition $4.1,\left(T_{F}\right)$ is a system of invariant integrals for $T(J)$ at $x$. Moreover, co orb ${ }_{T}(x)$ is weakly relatively compact so $\left(T_{F} x\right)$ has a weak limit point $y$. By Corollary 4.2 , $x \in X_{\text {erg }}(T)$.

Proposition 4.5. Let $T: J \rightarrow L(X)$ be a bounded representation. If $X$ is reflexive, or more generally if $N+R$ is dense in $X$, then $X_{\mathrm{erg}}=X$.

ProOF. Since $N+R \subseteq X_{\text {erg }} \subseteq X$ we conclude that $X_{\text {erg }}=X$ whenever $N+R$ is dense in $X$. It remains to prove that $N+R$ is dense in $X$ if $X$ is reflexive. For $S \subseteq X$ let $S^{\perp}=\left\{\varphi \in X^{*}:\langle x, \varphi\rangle=0\right.$ for all $\left.x \in S\right\}$. It is easy to check that $R^{\perp}=N\left(T^{*}\right)$. Hence for reflexive $X, R\left(T^{*}\right)^{\perp}=N\left(T^{* *}\right)=N$. Further, $N^{\perp}=R\left(T^{*}\right)^{\perp \perp}=\bar{R}\left(T^{*}\right)$. Hence $(N+R)^{\perp}=N^{\perp} \cap R^{\perp}=\bar{R}\left(T^{*}\right) \cap N\left(T^{*}\right)=\{0\}$, showing that $N+R$ is dense in $X$.

As an application we present the following

PROPOSITION 4.6. Given $A \in L(X)$ define $T: \mathbb{Z}^{+} \rightarrow L(X)$ by $T(n)=A^{n}$. If $x \in X_{\mathrm{erg}}(T)$ and $A^{n+1} x-A^{n} x \rightarrow 0$ as $n \rightarrow \infty$ then $A^{n} x \rightarrow y$ for some $y \in X$ with $A y=y$.

ProOF. We apply Theorem 2.7 with $\mathscr{A}=C_{0}(J, X), J=\mathbb{Z}^{+}$and $\varphi(n)=A^{n} x$. Since $\Delta_{t} \varphi \in \mathscr{A}$ for all $t \in J$ and $\varphi \in E(J, X)$ we conclude that $\varphi-M_{\varphi} \in \mathscr{A}$. So $A^{n} x \rightarrow y$ where $y=M_{\varphi}$. 
REMARK 4.7. If $A \in L(X)$ and $T: \mathbb{Z}^{+} \rightarrow L(X)$ is given by $T(n)=A^{n}$ then $N(T)=\operatorname{ker}(A-I)$ and $R(T)=\operatorname{range}(A-I)$. If $A$ is power bounded then $T$ is a bounded representation and if the Cesàro sums $A_{n} x=(1 / n) \sum_{j=1}^{n} A^{j} x$ converge weakly for some $x \in X$ then $T(\cdot) x$ is ergodic. If in addition $X$ is reflexive then by Propositions 4.1 and 4.5, $X=N \oplus \bar{R}$. This special case may be found in [20, p.214]. Also see [10].

\section{Cesàro and other means}

Throughout this section we will assume that $J$ is a measurable sub-semigroup of a locally compact abelian group $G$ carrying a fixed Haar measure $\mu$. Let $\mathscr{K}(G)$ denote the set of compact neighbourhoods of 0 in $G$ and set $\mathscr{K}(J)=\{V \cap J: V \in \mathscr{K}(G)$ and $\mu(V \cap J) \neq 0\}$. We shall call a net $\left(K_{\alpha}\right)_{\alpha \in \Lambda}$ in $\mathscr{K}(J)$, a Følner net if

$$
\lim _{\alpha \in \Lambda} \frac{\mu\left(K_{\alpha} \Delta\left(K_{\alpha}+s\right)\right)}{\mu\left(K_{\alpha}\right)}=0 \quad \text { for all } s \in J
$$

where $\Delta$ denotes symmetric difference.

Condition (5.1) was introduced by Følner (see [6, p.80]). As an example, let $G=\mathbb{R}^{2}$ and $J=\left\{\left(x_{1}, x_{2}\right) \in \mathbb{R}^{2}:\left|x_{2}\right| \leq m\left(x_{1}-a\right)\right\}$ where $a \geq 0$ and $m>0$. If $K_{r}=\{x \in J:|x| \leq r\}$ then $K_{r} \in \mathscr{K}(J), \mu(K) \sim r^{2}$ and $\mu\left(K_{r} \Delta\left(K_{r}+s\right)\right) \sim r$ for fixed $s \in J$. Hence $\left(K_{r}\right)_{r>a}$ is a Følner net.

We define the Cesàro integrals of functions $\varphi \in C_{b}(J, X)$ by $C_{K} \varphi(t)=(1 / \mu(K))$ $\int_{K} \varphi(t+s) d \mu(s)$ for $K \in \mathscr{K}(J), t \in J$.

PROPOSITION 5.1. If (5.1) holds then $\left(C_{K_{\alpha}}\right)_{\alpha \in \Lambda}$ is a system of invariant integrals for the translation semigroup $\mathscr{R}$ acting on $C_{u b}(J, X)$.

PROOF. Let $K \in \mathscr{K}(J)$ and $\varphi \in C_{u b}(J, X)$. Given $\varepsilon>0$ choose $V \in \mathscr{K}(G)$ such that $\left\|\varphi_{s}-\varphi_{r}\right\|_{\infty}<\varepsilon$ for all $t \in J$ and all $s \in(t+V) \cap J$. Since $\left\|C_{K} \varphi(s)-C_{K} \varphi(t)\right\| \leq$ $\left\|\varphi_{s}-\varphi_{t}\right\|_{\infty}$ we conclude that $C_{K} \varphi \in C_{u b}(J, X)$. Moreover, $C_{K} \in L\left(C_{u b}(J, X)\right)$. Next, by the compactness of $K$ we can choose $t_{1}, \ldots, t_{m} \in K$ such that $K \subseteq \bigcup_{j=1}^{m}\left(t_{j}+V\right)$. Set $\pi_{1}=\left(t_{1}+V\right) \cap K$ and for $2 \leq j \leq m, \pi_{j}=\left(t_{j}+V\right) \cap K \backslash \bigcup_{i=1}^{j-1} \pi_{j}$. Then $K=\bigcup_{j=1}^{m} \pi_{j}$ and the $\pi_{j}$ are disjoint measurable sets. Since

$$
\left\|C_{K} \varphi-\sum_{j=1}^{m} \frac{\mu\left(\pi_{j}\right)}{\mu(K)} \varphi_{t_{j}}\right\|<\varepsilon
$$

we conclude that $C_{K} \varphi \in \overline{\operatorname{co}} \operatorname{orb}_{\mathscr{R}}(\varphi)$, thereby proving (2.1). 
For (2.3), let $s \in J$. Then

$$
\begin{aligned}
\left\|\left(C_{K} R_{s}-C_{K}\right) \varphi\right\|_{\infty} & =\sup _{t \in J}\left\|\frac{1}{\mu(K)} \int_{K}[\varphi(t+s+u)-\varphi(t+u)] d \mu(u)\right\| \\
& =\sup _{t \in J}\left\|\frac{1}{\mu(K)} \int_{K \Delta(K+s)} \varphi(t+u) d \mu(u)\right\| \\
& \leq\|\varphi\|_{\infty} \frac{\mu(K \Delta(K+s))}{\mu(K)}
\end{aligned}
$$

and (2.3) follows from (5.1). Since (2.2) is clear, the proposition is proved.

COROLlARY 5.2. If $\varphi \in C_{u b}(J, X)$ and (5.1) holds, then the following are equivalent

(i) $\varphi \in E(J, X)$ and $M(\varphi)=y$,

(ii) the net $\left(C_{K_{\alpha}} \varphi\right)_{\alpha \in \Lambda}$ converges to $y$,

(iii) some subnet of $\left(C_{K_{\alpha}} \varphi\right)_{\alpha \in \Lambda}$ converges weakly to $y$.

PROOF. By Corollary 2.5 and Eberlein's mean ergodic theorem again, each of these conditions is equivalent to $y \in \overline{\mathrm{co}} \operatorname{orb}_{\mathscr{R}}(\varphi)$ with $y$ a constant function.

We come to our final system of invariant integrals. Let $\mathscr{P}=\left\{f \in L^{1}(G): f \geq 0\right.$ and $\hat{f}(0)=1\}$. Reiter [14, p.113] has proved the existence of a net $\left(f_{\alpha}\right)_{\alpha \in \Lambda}$ in $\mathscr{P}$ satisfying $\lim _{\alpha \in \Lambda}\left\|R_{s} f_{\alpha}-f_{\alpha}\right\|_{1}=0$ for all $s \in G$. For $\varphi \in C_{u b}(G, X)$ we can define $A_{\alpha} \varphi \in C_{u b}(G, X)$ by $A_{\alpha} \varphi=\varphi * f_{\alpha}$. So $\left\|A_{\alpha} \varphi\right\|_{\infty} \leq\|\varphi\|_{\infty}$ and $A_{\alpha} \in L\left(C_{u b}(G, X)\right)$.

PROPOSITION 5.3. The net $\left(A_{\alpha}\right)_{\alpha \in \Lambda}$ is a system of invariant integrals for the translation semigroup $\mathscr{R}=\left(R_{s}\right)_{s \in G}$ acting on $C_{u b}(G, X)$.

PROOF. Given $V \in \mathscr{K}(G)$ and $\varphi \in C_{u b}(G, X)$ let $f_{V}=(1 / \mu(V)) \chi_{-V}$ where $\chi_{-V}$ is the characteristic function of $-V$. Then $f_{V} \in \mathscr{P}$ and since $\varphi * f_{V}=$ $(1 / \mu(V)) \int_{V} \varphi_{s} d \mu(s)=C_{V} \varphi$, it follows from Proposition 5.1 that $\varphi * f_{V} \in \overline{\operatorname{co}} \operatorname{orb}_{\mathscr{R}}(\varphi)$. It is easy to check that $\mathscr{P} \subseteq \overline{\mathrm{co}}\left\{f_{V}: V \in \mathscr{K}(G)\right\}$. Hence, $\varphi * \mathscr{P} \subseteq \overline{\mathrm{co}}$ orb $\mathscr{R}(\varphi)$, proving (2.1). Since $\left\|A_{\alpha}\right\| \leq 1,(2.2)$ holds. Finally, for $s \in G$ we have $\left\|\left(A_{\alpha} R_{s}-A_{\alpha}\right) \varphi\right\|_{\infty}=\left\|\left(R_{s} \varphi-\varphi\right) * f_{\alpha}\right\|_{\infty}=\left\|\varphi *\left(R_{s} f_{\alpha}-f_{\alpha}\right)\right\|_{\infty} \leq\|\varphi\|_{\infty}\left\|R_{s} f_{\alpha}-f_{\alpha}\right\|_{1}$.

From the definition of $\left(f_{\alpha}\right),(2.3)$ follows and the proposition is proved.

As for Corollary 5.2 we obtain

COROLlaRY 5.4. For $\varphi \in C_{u b}(G, X)$ the following are equivalent 
(i) $\varphi \in E(G, X)$ and $M(\varphi)=y$,

(ii) the net $\left(A_{\alpha} \varphi\right)_{\alpha \in \Lambda}$ converges to $y$,

(iii) some subnet of $\left(A_{\alpha} \varphi\right)_{\alpha \in \Lambda}$ converges weakly to $y$.

Argabright [2] used the Reiter nets $\left(f_{\alpha}\right)$ to prove an ergodic limit for scalar-valued Eberlein weakly almost periodic functions on $G$. Datry and Muraz [7] also used them to introduce ergodicity in Banach $L^{1}(G)$-modules.

We conclude with two more examples, firstly of some ergodic functions and secondly of a non-ergodic one. Recall that for a function $\varphi \in C_{b}(G, X)$ the set $I(\varphi)=\left\{f \in L^{1}(G): \varphi * f=0\right\}$ is a closed ideal of $L^{1}(G)$. Let $\widehat{G}$ denote the character group of $G, 0$ the unit of $\widehat{G}$, and $\hat{f}: G \rightarrow \mathbb{C}$ the Fourier transform of $f$. The Beurling spectrum of $\varphi$ is $\operatorname{sp}(\varphi)=\{\gamma \in \widehat{G}: \hat{f}(\gamma)=0$ for all $f \in I(\varphi)\}$.

THEOREM 5.5. If $\varphi \in C_{u b}(G, X)$ and $0 \notin \operatorname{sp}(\varphi)$ then $\varphi \in E(G, X)$.

Proof. Take $V \in \mathscr{K}(\widehat{G})$ with $V \cap \operatorname{sp}(\varphi)=\emptyset$ and $f \in L^{1}(G)$ with $\hat{f}(0)=1$ and $\operatorname{supp}(\hat{f}) \subseteq V$. Then $\operatorname{sp}(\varphi * f)=\emptyset$ so $\varphi * f=0$. Moreover, $f$ is continuous. Now, given $\varepsilon>0$, choose a compact set $K$ in $G$ such that $\int_{G \backslash K}|f(t)| d \mu(t)<$ $\varepsilon /\left(1+2\|\varphi\|_{\infty}\right)$. For $s \in G$ define $g(s)=\left(\varphi-\varphi_{-s}\right) f(s)$. Hence $\int_{G} g(s) d \mu(s)=$ $\varphi-\varphi * f=\varphi$. Moreover, by Proposition 2.2, $g(s) \in E(G, X)$ and since $\varphi$ is uniformly continuous, $g: G \rightarrow E(G, X)$ is continuous. Since $K$ is compact, $\left.g\right|_{K}$ is separably-valued and hence Bochner integrable. Therefore $\int_{K} g(s) d \mu(s) \in E(G, X)$. But $\left\|\varphi-\int_{K} g(s) d \mu(s)\right\| \leq\left\|\int_{G \backslash K} g(s) d \mu(s)\right\|<\varepsilon$ and $\operatorname{so} \varphi \in E(G, X)$ as claimed.

EXAMPLE 5.6. Define $\varphi: \mathbb{R} \rightarrow c_{0}$ by $\varphi(t)=(\sin (t / n))_{n=1}^{\infty}$. One easily checks that $\varphi \in C_{u b}\left(\mathbb{R}, c_{0}\right)$. Now the range of $\varphi$ is not relatively compact in $c_{0}$. For, if it were, then

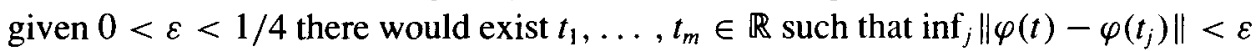
for all $t \in \mathbb{R}$. In particular we would have $|\sin (t / n)|<2 \varepsilon$ for all $n>N(\varepsilon)$ and all $t \in \mathbb{R}$, which is false. It follows that $\varphi$ is not almost periodic. On the other hand $\varphi^{\prime}$ is almost periodic (see [1, p. 53]) and so $\varphi \notin E\left(\mathbb{R}, c_{0}\right)$. For otherwise, by Levitan [13] or Basit [3, Theorem 3.1.1] it would follow that $\varphi$ is almost periodic. From Theorem 5.5 we conclude that $0 \in \operatorname{sp}(\varphi)$.

\section{References}

[1] L. Amerio and G. Prouse, Almost periodic functions and functional equations (Van Nostrand, New York, 1971).

[2] L. N. Argabright, 'On the mean value of weakly almost periodic functions', Proc. Amer. Math. Soc. 36 (1972), 315-316.

[3] B. Basit, 'Some problems concerning different types of vector-valued almost periodic type functions', Dissertationes Math. (Rozprawy Mat.) 338 (1995). 
[4] - 'Harmonic analysis and asymptotic behavior of solutions to the abstract Cauchy problem', Semigroup Forum 54 (1997), 58-74.

[5] B. Basit and A. J. Pryde, 'Polynomials and functions with finite spectra on locally compact abelian groups', Bull. Austral. Math. Soc. 51 (1994), 33-42.

[6] J. F. Berglund, H. D. Junghenn and P. Milnes, Analysis on Semigroups: Function spaces, compactifications, representations (Wiley-Interscience, New York, 1989).

[7] C. Datry and G. Muraz, 'Analyse harmonique dans les modules de Banach II: presque-périodicité et ergodicité', Bull. Science Math. (2) 120 (1996), 493-536.

[8] N. Dunford and J. T. Schwartz, Linear operators, Parts I, II (Interscience, New York, 1958, 1963).

[9] W. F. Eberlein, 'Abstract ergodic theorems and weak almost periodic functions', Trans. Amer. Math. Soc. 69 (1949), 217-240.

[10] J. A. Goldstein, 'Application of operator semigroups to Fourier analysis', Semigroup Forum 52 (1996), 37-47.

[11] K. Iseki, 'Vector valued functions on semigroups, I-III', Proc. Japan Acad. Ser. A Math. Sci. (1955), 16-19, 152-155 and 699-702.

[12] Y. Katznelson and L. Tzafriri, 'On power bounded operators', J. Funct. Anal. 68 (1986), 313-328.

[13] B. M. Levitan, 'Integration of almost periodic functions with values in Banach spaces', Math. USSR-Izv. 30 (1966), 1101-1110 (in Russian).

[14] H. Reiter, Classical Fourier analysis on locally compact groups (Oxford University Press, 1968).

[15] W. Rudin, Harmonic analysis on groups (Interscience, New York, 1963).

[16] W. M. Ruess and V. Q. Phóng, 'Asymptotically almost periodic solutions of evolution equations in Banach spaces', J. Differential Equations 122 (1995), 282-301.

[17] W. M. Ruess and W. H. Summers, 'Weak almost periodicity and the strong ergodic limit theorem for contraction semigroups', Israel J. Math. 64 (1988), 139-157.

[18] —, 'Integration of asymptotically almost periodic functions and weak almost periodicity', Dissertationes Math. (Rozprawy Mat.) 279 (1989).

[19] - 'Ergodicity theorems for semigroups of operators', Proc. Amer. Math. Soc. 114 (1992), 423-432.

[20] K. Yosida, Functional Analysis (Springer, Berlin, 1966).

[21] C. Zhang, 'Vector-valued means and their applications in some vector-valued function spaces', Dissertationes Math. (Rozprawy Mat.) 334 (1994).

\section{Department of Mathematics}

Monash University

Clayton, VIC 3168

Australia

e-mail: bbasit(ajpryde)@vaxc.cc.monash.edu.au 